\title{
Timing and Efficacy of 595-nm Pulsed-Dye Laser Combined with $0.5 \%$ Timolol Maleate Solution in the Treatment of Superficial Infantile Hemangiomas
}

\section{Weikang Shi $\mathbb{D}^{*}$ \\ Huiyi He* \\ Jicong Jiang (D) \\ Yu Gao \\ Hehe Quan}

Department of Dermatology, The Second Affiliated Hospital and Yuying Children's Hospital of Wenzhou Medical University, Wenzhou, 325027, Zhejiang Province, People's Republic of China

*These authors contributed equally to this work
Correspondence: Hehe Quan Email 13587748556@qq.com
Purpose: Infantile hemangioma $(\mathrm{IH})$ is the most common benign tumor in infancy, and superficial IH is the most common type. IH can reportedly resolve spontaneously, but this is associated with complications, such as scars, atrophy, hypopigmentation, telangiectasia, and skin sagging, in $70 \%$ of cases. This study explores the safety and feasibility of therapeutic intervention with the 595-nm pulsed-dye laser (PDL) combined with $0.5 \%$ timolol maleate solution in superficial IH and compares the difference in efficacy between the early group and the late group.

Patients and Methods: This retrospective study examined 167 patients with superficial IH who underwent combination therapy at the Dermatology Clinic of the Second Affiliated Hospital and Yuying Children's Hospital of Wenzhou Medical University between July 2019 and July 2020. The early and late groups were composed of children aged $\leq 2$ and $>2$ months, respectively. Treatment was administered for 6 months, and patients were followed up for another 6 months. Two independent, double-blinded physicians reviewed photographs of the skin lesions before and after treatment to evaluate efficacy.

Results: The early group demonstrated higher treatment efficacy than the late group. The early $(n=45)$ and late $(n=122)$ groups had treatment efficacy rates of $95.5 \%$ and $86.1 \%$, respectively; the difference was statistically significant $(P<0.05)$. The early and late groups underwent $3.51 \pm 0.50$ and $4.73 \pm 0.68$ months of treatment, respectively; the difference was statistically significant $(P<0.05)$. Seventeen $(44.4 \%)$ patients in the early group had immediate adverse reactions but no permanent sequelae, whereas $25(20.49 \%)$ and $13(10.7 \%)$ patients in the late group had immediate and permanent sequelae, respectively. The difference was statistically significant $(P<0.05)$.

Conclusion: This retrospective study demonstrated that 595-nm PDL combined with $0.5 \%$ timolol maleate solution was a safe and effective local treatment for superficial IH. Early treatment required fewer treatments, had better curative effects, and a lower probability of permanent sequelae.

Keywords: infantile hemangiomas, pulsed-dye laser, timolol maleate solution, timing and efficacy

\section{Introduction}

Infantile hemangioma (IH) is the most common benign tumor in infancy. IH is observed in approximately $4-5 \%$ of mature neonates and is $2.3-2.9$ times more common in female and white patients. ${ }^{1}$ Premature birth and low weight are also risk factors. ${ }^{2}$ IH can be subclassified based on the depth of involvement. The superficial type is the most common and accounts for approximately $50-60 \%$ of all cases, whereas the deep and mixed types 
affect $15 \%$ and $25-35 \%$ of patients, respectively. While IH demonstrates differences in the depth of involvement, it evolves in a characteristic pattern that involves proliferation, stabilization, and slow, spontaneous involution. ${ }^{3}$ Some scholars recommend the "wait and see" strategy for all patients, except for those with severe complications that require treatment, ${ }^{4}$ because the majority of $\mathrm{IH}$ resolves spontaneously. However, approximately $70 \%$ of children who are managed with this approach demonstrate degenerative skin and subcutaneous tissue changes, such as scars, atrophy, hypopigmentation, telangiectasia, and skin relaxation. ${ }^{5}$ Moreover, over $60 \%$ of IH occur in the head and neck. ${ }^{6}$ If IH is left untreated, the residual skin lesions may result in significant physical and psychological sequelae in the children and their parents. Some studies have demonstrated that the severity of skin damage is related to the final size of the tumor and degree of involvement of the subcutaneous structures. ${ }^{7}$ Early treatment of IH, before it enlarges and thickens, may reduce the incidence of skin damage. Topical timolol solution and pulsed-dye laser (PDL) have shown good safety and efficacy in controlling the growth and promoting the regression of superficial $\mathrm{IH} ;{ }^{8}$ however, only a few clinical trials have compared the efficacy of combined therapy hemangiomas. This study examined the safety and efficacy of 595-nm PDL combined with topical timolol solution for superficial IH and compared the efficacy of this method for early and late hemangiomas.

\section{Patients and Methods}

\section{Participants}

This study was conducted in accordance with the Declaration of Helsinki and approved by the independent ethics committee of the Second Affiliated Hospital and Yuying Children's Hospital of Wenzhou Medical University. Written informed consent was obtained from the patients' guardians. Patients with superficial IH were divided into the early ( $\leq 2$ months, $\mathrm{n}=45)$ and late $(>2$ months, $\mathrm{n}=122$ ) groups according to age. The early group had 24 females and 21 males aged 20-60 days (mean, 48.2 $\pm 2.64 \mathrm{~d}$ ). The late group had 62 females and 60 males aged 2-12 months (mean, $6.25 \pm 1.18 \mathrm{~m}$ ). There were no significant differences in the sex and location of the hemangiomas between the two groups $(P<0.05)$ (Table 1).

\section{Inclusion and Exclusion Criteria}

The inclusion criteria were as follows: (1) patients newly diagnosed with superficial IH according to the classification and diagnostic criteria of the International Vascular
Table I Baseline Characteristics at Time of Inclusion

\begin{tabular}{|l|l|l|}
\hline Characteristic & $\begin{array}{l}\text { Early Treatment } \\
\text { Group, } \mathbf{n = 4 5}\end{array}$ & $\begin{array}{l}\text { Late Treatment } \\
\text { Group, } \mathbf{n = ~ I 2 2}\end{array}$ \\
\hline Sex, n (\%) & $24(53.3 \%)$ & $60(49.3 \%)$ \\
\hline Male & $21(46.7 \%)$ & $62(50.7 \%)$ \\
\hline Female & & \\
\hline Location, $\mathrm{n}(\%)$ & $12(26.7 \%)$ & $51(41.8 \%)$ \\
\hline Head and face & $15(33.3 \%)$ & $37(30.3 \%)$ \\
\hline Trunk & $18(40.0 \%)$ & $34(27.9 \%)$ \\
\hline Limbs & $1.61 \pm 0.09$ & $6.25 \pm 1.18$ \\
\hline $\begin{array}{l}\text { Average age at first visit } \\
\text { (months, } \bar{x}+1.96 \mathrm{~s})\end{array}$ & & \\
\hline
\end{tabular}

Anomaly Research Association for vascular diseases and the thickness of the tumor is less than $3 \mathrm{~mm}$; (2) no prior treatment; (3) normal cardiac auscultation, electrocardiogram, and cardiac color Doppler ultrasound findings before treatment; (4) good patient's guardian compliance; and (5) able to follow-up for at least 6 months. The exclusion criteria were as follows: (1) received other treatments in the past or during the treatment period; (2) low compliance and poor adherence to the treatment; (3) deep and multiple hemangiomas; and (4) co-morbidities, such as bronchial asthma, pneumonia, heart block, severe or acute heart failure, or sinus bradycardia.

\section{Treatment Methods}

The Vbeam Perfecta (Candela Medical, Wayland, MA, USA) was applied with the following settings: wavelength, $595 \mathrm{~nm}$; energy density, 6.0-7.5 J/cm2; spot diameter,5$10 \mathrm{~mm}$; pulse width, $0.45-40 \mathrm{~ms}$, and dynamic cooling device (DCD) spray, 30-40 ms, and interval 20-30 ms. The risks and prognosis were communicated to the family before treatment. An informed consent form was signed, and preprocedural pictures were taken. The laser parameters were selected according to the area, color, and thickness of the lesion and adjusted according to the lesion's immediate response. The laser was applied until the skin lesions became grayish white or dark purple in color. Protective goggles were worn by both the child and operator during the treatment. After treatment, medical dressing and a cold compress was applied to the treated area for $20 \mathrm{~min}$. Fusidic acid cream was prescribed for 7 days to prevent infection. Laser treatment was performed 
at 4-week intervals, and the treatment time was determined by individual patient conditions. Timolol maleate solution $0.5 \% 5 \mathrm{mg} / \mathrm{mL}$ (Wuhan Wujing Pharmaceutical Co., Ltd., National Medicine Standard H42021078) was instilled on absorbent cotton $(30-40 \mathrm{ul} / \mathrm{cm} 2)$ and applied to the lesion thrice daily for $30 \mathrm{~min}$. The cotton gauze was secured with plastic wrap. The guardians were advised to observe for localized redness, erosion, ulceration, and changes in the child's complexion, breathing, and heart rate after each application. The guardians recorded the child's heart rate during rest or sleep before and after the medication was administered. If significant changes in the heart rate were noted, the guardians were advised to stop the treatment immediately and seek hospital consult. The guardians were also advised to avoid contact between the medication and eyes or mucus membranes of the genital tract.

\section{Documentation of Adverse Reactions During Follow-Up}

After treatment cessation, patients were followed up monthly for 6 months. The incidence of localized skin redness, itching, erosions, ulcers, hyperpigmentation, hypopigmentation, residual fibrofatty tissue, and changes in skin texture, such as scars, were recorded. The incidence of systemic adverse reactions, such as bradycardia, shortness of breath, asthma, and sleep disorders, were also recorded.

\section{Evaluation of Efficacy}

Digital photographs of the lesions were taken before each treatment and 6 months after the first treatment. A digital camera was used under the same conditions to record the occurrence of adverse reactions, such as blisters, pigmentary changes, and scar formation. Two independent, doubleblinded doctors reviewed the photographs to determine the degree of clinical improvement and evaluate the therapeutic effect. Six months after the first treatment, the overall regression of the hemangioma was evaluated using the standard grading proposed by Achauer et al. Grade I (invalid) regression was defined by tumor reduction of $<25 \%$, no obvious change in color, no reduction in the tumor echo signal, and high blood flow on color Doppler ultrasound examination; Grade II (effective) regression was defined by tumor reduction of $25-50 \%$, color gradually becomes lighter, resolution of maximum abnormal blood flow on color Doppler ultrasound examination; Grade III (marked effect) regression was defined by tumor reduction of $>51 \%$, the color is visibly lighter, and marked resolution of abnormal blood flow signals on color Doppler ultrasound examination; and Grade IV (healed) regression was defined by tumor reduction of $>75 \%$, the color basically disappears, and complete resolution of the abnormal blood flow signals on color Doppler ultrasound examination. The efficacy rate was calculated as the total sum of cured cases and markedly effective cases divided by the total number of cases $\times 100 \%$ (Figure 1).

\section{Statistical Analysis}

Statistical analysis was performed with SPSS version 26.0 (IBM, Armonk, NY, USA), The Mann-Whitney test was performed to evaluate the efficacy of the treatment and treatment duration, whereas the Chi-square test was performed to analyze adverse reactions. A $P$-value of $<0.05$ was considered statistically significant.

\section{Results}

\section{Efficacy}

The early group included 45 cases of superficial IH. 1, 1, 12, and 31 cases demonstrated grade I, II, III, and IV regression, respectively. The efficacy rate in the early group was $95.5 \%$. In comparison, the late group included 122 children.7,10, 42, and 63 cases demonstrated grade I, II, III, and IV regression, respectively. The efficacy rate in the late group was $86.1 \%$. The efficacy rate in the early group was significantly higher than in the late group $(P=$ 0.031) (Table 2).

\section{Treatment Time}

The early and late groups underwent $3.51 \pm 050$ and 4.73 \pm 0.68 months of treatment sessions, respectively. The treatment group demonstrated faster results and shorter treatment time than the control group. The difference was statistically significant $(P<0.001)$ (Table 3$)$.

\section{Adverse Reactions}

In the early group, 17 (37.78\%) patients developed various degrees of blisters and scabs, which were effectively treated with topical fusidic acid cream over 1 week. Three (6.67\%) patients demonstrated hyperpigmentation, but the hyperpigmentation subsided spontaneously within 2 months of treatment end. There were no long-term complications, such as infection and scars, as well as no adverse cardiovascular, respiratory, eye, or systemic reactions. 


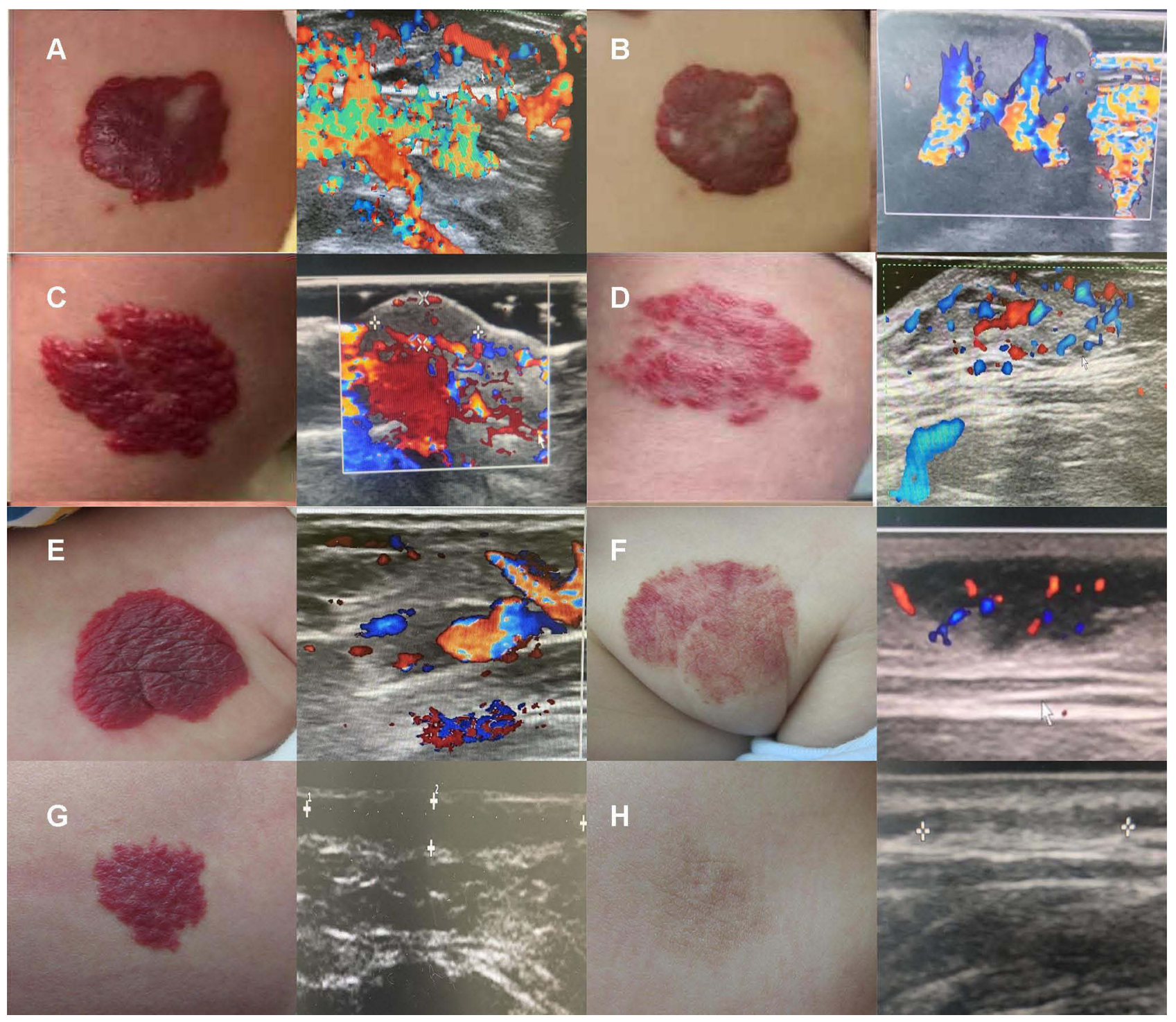

Figure I Images of before and after treatment and evaluation of efficacy.(A and B) Grade I (invalid) regression was defined by tumor reduction of $<25 \%$, no obvious change in color, no reduction in the tumor echo signal, and high blood flow on color Doppler ultrasound examination; (C and D) Grade II (effective) regression was defined by tumor reduction of $25 \%-50 \%$, color gradually becomes lighter, resolution of maximum abnormal blood flow on color Doppler ultrasound examination; (E and $\mathbf{F}$ ) Grade III (marked effect) regression was defined by tumor reduction of $>51 \%$, the color is visibly lighter, and marked resolution of abnormal blood flow signals on color Doppler ultrasound examination; and $(\mathbf{G}$ and $\mathbf{H})$ Grade IV (healed) regression was defined by tumor reduction of $>75 \%$, the color basically disappears, and complete resolution of the abnormal blood flow signals on color Doppler ultrasound examination.

In the late group, 25 (20.49\%) patients had blisters and scabs of varying degrees. These were also effectively treated with topical fusidic acid cream over one week and were not associated with complications, such as infection and scarring. Post- treatment pigmentation occurred in 14 (11.47\%) cases; 10 (8.19\%) cases resolved within 2 months of the end of treatment, but four $(2.56 \%)$ cases persisted. Seven (5.73\%) patients demonstrated

Table 2 Comparison of Efficacy for Two Groups [n(\%)]

\begin{tabular}{|l|l|l|l|l|l|}
\hline \multirow{2}{*}{ Classify } & \multicolumn{5}{|c|}{ Efficacy Rank } \\
\cline { 2 - 6 } & I & II & III & IV & Total(\%) \\
\hline Early treatment group & I (2.2) & I (2.2) & I2 (26.7) & $31(68.9)$ & 95.5 \\
\hline Late treatment group & $7(5.7)$ & $10(8.2)$ & $42(34.4)$ & $63(51.7)$ & 86.1 \\
\hline
\end{tabular}


Table 3 Comparison of Treatment Time for Two Groups

\begin{tabular}{|l|c|c|}
\hline Treatment Time & $\begin{array}{c}\text { Early Treatment } \\
\text { Group (n=45) }\end{array}$ & $\begin{array}{c}\text { Late Treatment } \\
\text { Group (n= 122) }\end{array}$ \\
\hline One month & 8 & 2 \\
\hline Two months & 4 & 22 \\
\hline Three months & 12 & 6 \\
\hline Four months & 7 & 7 \\
\hline Five months & 6 & 25 \\
\hline Slx months & 8 & 60 \\
\hline $\begin{array}{l}\text { Average treatment } \\
\text { time }(\bar{x}+1.96 \mathbf{s})\end{array}$ & $3.51 \pm 0.50$ & $4.73 \pm 0.68$ \\
\hline
\end{tabular}

fibrofatty scars and skin sagging, whereas six (4.91\%) patients had dry desquamation and localized itching. No adverse cardiovascular, respiratory, eye, or systemic reactions were noted. The difference was statistically significant $(P<0.027$ (Table 4).

\section{Discussion}

IH remains one of the most common vascular tumors among infants. Superficial IH is the predominant type, which may subside spontaneously. The current recommended approach is to "wait and see," but this may result in permanent complications. ${ }^{9} \mathrm{IH}$ naturally gradually subsides after 1 year of age. However, while some IHs resolve spontaneously, there remains a risk of complications, such as ulcers, scars, and functional deficit. Approximately $60 \%$ of all $\mathrm{IHs}$ are located in the face. As the IH grows, parents often experience increasing long-term anxiety ${ }^{10}$ and are more likely to desire early treatment to prevent or reduce potential pathologic and psychologic complications. IH is also more likely to subside with residual lesions, and the severity of the residual lesions is related to the final size of the $\mathrm{IH}^{7}$ Therefore, it is extremely important to control tumor growth as early and effectively as possible. $^{11}$

At present, there are several treatment methods for IH, including oral propranolol, hormone therapy, surgery, cryotherapy, and laser treatment. Oral propranolol was first reported as an effective treatment for IH in $2008 .{ }^{12}$ It is currently the first-line medical option for $\mathrm{IH}$, but studies have identified systemic risks associated with oral propranolol, such as hypotension, bradycardia, and bronchospasm. Oral propranolol also demonstrates first-pass liver effects, which result in poor bioavailability. ${ }^{13}$ As such, oral propranolol is not suitable for superficial IH. Topical timolol is often used in the clinical setting; however, its exact mechanism of action remains unclear. Data suggest that timolol constricts blood vessels within the first 3 days of treatment. In the mid-term, it blocks angiogenic factors, such as vascular endothelial growth factor, fibroblast growth factors, matrix metalloproteinases, and low $\mathrm{pH}$, which arrest the growth of the hemangioma. In the longterm effect, it induces endothelial cell apoptosis, which results in tumor regression. ${ }^{14}$ Studies have demonstrated that topical timolol is effective and safe for superficial $\mathrm{IH}^{15,16}$ A multicenter retrospective cohort study analyzed 731 patients who received topical timolol for IH. Topical timolol provided the best response in patients with superficial IH with $<1 \mathrm{~mm}$ depth, and only $3.4 \%$ of these patients experienced adverse events. ${ }^{17}$ According to a systematic review, the response rate of $\mathrm{IH}$ to topical timolol is as high as $83 \%$, and adverse events are rare. $^{18}$

Compared with the risks with anesthesia and surgery, particularly surgical scars, laser treatment is a safe and noninvasive option for IH. The target chromophore for laser treatment in IH is oxygenated hemoglobin. Oxyhemoglobin absorbs light to generate heat. The heat is transferred to the walls of the surrounding blood vessels and prompt damage. ${ }^{19}$ PDL, an Nd:YAG laser, and the dual-wavelength laser have been utilized to treat IH. PDL, which has a wavelength of 595 $\mathrm{nm}$, is more widely used. The most common side effects of PDL include purpura, swelling, skin atrophy, and hypopigmentation. Compared with the traditional PDL, the 595-nm PDL is equipped with a DCD that reduces pain and prevents damage to the surrounding healthy tissue. This greatly reduces the likelihood for adverse reactions, such as skin atrophy and pigmentation. ${ }^{20}$ Many studies have demonstrated that the 595$\mathrm{nm}$ PDL is an effective treatment for superficial IH. ${ }^{8,21}$ Rizzo et al reported efficacy rates as high as $81 \%$ in the absence of adverse events, such as scars or tissue atrophy. ${ }^{22}$ However, the

Table 4 Comparison of Complications for Two Groups

\begin{tabular}{|l|l|l|l|l|l|}
\hline Classify & Blister & Pigmentation & Scar & Scales & Total(n, \%) \\
\hline Early treatment group & 17 & 3 & 0 & 0 & $20(44.4)$ \\
\hline Late treatment group & 25 & 14 & 7 & 6 & $52(42.6)$ \\
\hline
\end{tabular}


595-nm PDL is not very effective for deep or thick IH. The deep components of these lesions may continue to proliferate even after laser treatment. ${ }^{23}$ PDL only penetrates to a depth of 1-2 mm, which corresponds with $1 / 10$ of the thickness of some $\mathrm{IH}^{24}$ Compared with the 595-nm PDL, the 1064-nm laser is more effective for deeper $\mathrm{IH}$, but the incidence of adverse events is also higher. ${ }^{20}$ Therefore, we believes that there is a greater benefit to treating superficial IH early than just waiting and seeing.

Both PDL and topical timolol have shown good efficacy and safety profiles in superficial $\mathrm{IH}$, and some studies have proposed that combined treatment is even more effective, requires shorter treatment duration, and has fewer adverse effects. ${ }^{25}$ Only a few clinical trials have examined combined treatment in early and late IH. Our study analyzed the safety and efficacy of combined therapy in the treatment of superficial $\mathrm{IH}$ and compare the difference in efficacy between the early group and the late group.

Our data demonstrated an $86.1 \%$ efficacy rate in the late treatment group, which was not significantly different from the results of the meta-analysis on combined treatment conducted by Chen et al. In comparison, the efficacy rate in the early treatment group was $95.5 \%$, which was significantly higher than that of the late group. Compared with topical timolol or PDL laser treatment alone, the combined treatment had a higher efficacy rate, which suggested that combined treatment is especially effective for the early treatment of superficial IH.

In this study, the early and late groups underwent $3.51 \pm$ 0.50 , and $4.73 \pm 0.68$ treatments, respectively. The early treatment group underwent statistically significantly less treatments than the late treatment group $(P<0.05)$. Hunzeker et al Sometreated IH with PDL alone, which required an average of 5.6 treatments to achieve an efficacy rate of $77.3 \%{ }^{26}$ Combined treatment has obvious advantages. Some scholars have proposed that synergy may improve the curative effect of combination therapy. PDL treatment utilizes a certain wavelength of laser light, which is selectively absorbed by the oxygenated hemoglobin in the diseased blood vessel, resulting in targeted destruction of diseased vascular tissue. Concurrently, $0.5 \%$ timolol maleate solution is applied over the hemangioma, which prompts further blood vessel and tumor atrophy during laser treatment. Combination therapy provides a synergistic effect that results in rapid and effective effects. $^{21}$

The early group did not demonstrate any significant adverse effects, except for immediate blisters and a small amount of pigmentation. These adverse reactions were temporary and resolved within two weeks; however, the early treatment group had a higher incidence of blisters than the late treatment group. We believe that this may be due to the more delicate skin of newborns. Smaller energy sources should be utilized in treatment during early childhood. The majority of adverse reactions in the late treatment group resolved over time, but residual fibrofatty scars and skin laxity, which are difficult to treat, were noted in approximately $6 \%$. These adverse effects may be due to the long interval between disease onset and treatment or the large size of the hemangiomas. However, regardless of whether early or late treatment was administered, the incidence of complications following treatment was significantly lower than the incidence of complications following spontaneous regression (70\%). Combination treatment can also reduce the required laser parameters, such as energy size and pulse time, which reduces the risk of complications, such as blisters, purpura, bleeding, and infection. ${ }^{27}$

\section{Limitations}

This study has some limitations. First, our treatment endpoint was at 6 months, but some patients required more treatments to achieve better results. Second, the follow-up time of this study was also only 6 months. We did not evaluate the long-term efficacy of the treatment and may have missed long-term sequelae and disease recurrence. Meanwhile the mechanism of the better treatment effect of laser combined with topical timolol in children younger than 2 months and whether it is related to intervention before or early proliferation of hemangioma remains to be further basic research.

\section{Conclusion}

This retrospective study demonstrated that 595-nm PDL combined with $0.5 \%$ timolol maleate solution was effective and safe for the local treatment of superficial IH. Early treatment of IH was associated with fewer treatments, better curative effects, and a lower risk for sequelae. 595$\mathrm{nm}$ PDL combined with timolol maleate solution shows strong promise for the treatment of superficial IH in children in the clinical setting. In summary, recent research has demonstrated that PDL, $\beta$-receptor blockers, or both effectively treat $\mathrm{IH}$ and may be an ideal method for the early treatment of superficial IH.

\section{Acknowledgments}

This study is supported by the National Natural Science Foundation of China (81801862). 


\section{Disclosure}

The authors report no conflicts of interest for this work.

\section{References}

1. Munden A, Butschek R, Tom W, et al. Prospective study of infantile haemangiomas: incidence, clinical characteristics and association with placental anomalies. Br J Dermatol. 2014;170(4):907-913. doi:10.1111/bjd.12804

2. Goelz R, Poets C. Incidence and treatment of infantile haemangioma in preterm infants. Arch Dis Child Fetal Neonatal Ed. 2015;100(1): F85-F91. doi:10.1136/archdischild-2014-306197

3. Chang L, Haggstrom A, Drolet B, et al. Growth characteristics of infantile hemangiomas: implications for management. Pediatrics. 2008;122(2):360-367. doi:10.1542/peds.2007-2767

4. Hoeger P, Harper J, Baselga E, et al. Treatment of infantile haemangiomas: recommendations of a European expert group. Eur J Pediatr. 2015;174(7):855-865. doi:10.1007/s00431-015-2570-0

5. Bauland C, Lüning T, Smit J, Zeebregts C, Spauwen P. Untreated hemangiomas: growth pattern and residual lesions. Plast Reconstr Surg. 2011;127(4):1643-1648. doi:10.1097/PRS.0b013e318208d2ac

6. Léauté-Labrèze C, Prey S, Ezzedine K. Infantile haemangioma: part I. Pathophysiology, epidemiology, clinical features, life cycle and associated structural abnormalities. J Eur Acad Dermatol Venereol. 2011;25(11):1245-1253. doi:10.1111/j.1468-3083.2011.04102.x

7. Enjolras O, Mulliken J. The current management of vascular birthmarks. Pediatr Dermatol. 1993;10(4):311-313. doi:10.1111/ j.1525-1470.1993.tb00393.x

8. Chen W, Liu S, Yang C, Yang S. Clinical efficacy of the $595 \mathrm{~nm}$ pulsed dye laser in the treatment of childhood superficial hemangioma - analysis of 10-year application in Chinese patients. J Dermatolog Treat. 2015;26 (1):54-58. doi:10.3109/09546634.2013.806979

9. Chelleri C, Monzani N, Gelmetti C, et al. Residual lesions after pharmacological and dye-laser treatment of infantile hemangiomas: critical review of 432 cases. Lasers Surg Med. 2020;52(7):597-603. doi:10.1002/lsm.23205

10. Moyakine A, Spillekom-van Koulil S, van der Vleuten C. Propranolol treatment of infantile hemangioma is not associated with psychological problems at 7 years of age. $J$ Am Acad Dermatol. 2017;77(1):105-108. doi:10.1016/j.jaad.2017.01.025

11. Moyakine A, Hermans D, Fuijkschot J, van der Vleuten C. Propranolol treatment of infantile hemangiomas does not negatively affect psychomotor development. J Am Acad Dermatol. 2015;73 (2):341-342. doi:10.1016/j.jaad.2015.04.053

12. Léauté-Labrèze C, Dumas de la Roque E, Hubiche T, Boralevi F, Thambo J, Taïeb A. Propranolol for severe hemangiomas of infancy. N Engl J Med. 2008;358(24):2649-2651. doi:10.1056/NEJMc0708819

13. Cheng C, Friedlander S. Infantile hemangiomas, complications and treatments. Semin Cutan Med Surg. 2016;35(3):108-116. doi:10.1 2788/j.sder.2016.050

14. Calvo M, Garcia-Millán C, Villegas C, Fueyo-Casado A, Burón I. Topical timolol for infantile hemangioma of the eyelid. Int J Dermatol. 2013;52(5):603-604. doi:10.1111/j.1365-4632.20 11.05290.x

Clinical, Cosmetic and Investigational Dermatology

Publish your work in this journal

Clinical, Cosmetic and Investigational Dermatology is an international, peer-reviewed, open access, online journal that focuses on the latest clinical and experimental research in all aspects of skin disease and cosmetic interventions. This journal is indexed on CAS.
15. Püttgen K, Lucky A, Adams D, et al. Topical timolol maleate treatment of infantile hemangiomas. Pediatrics. 2016;138(3). doi:10.1542/peds.2016-0355

16. Frommelt P, Juern A, Siegel D, et al. Adverse events in young and preterm infants receiving topical timolol for infantile hemangioma. Pediatr Dermatol. 2016;33(4):405-414. doi:10.1111/pde.12869

17. Chakkittakandiyil A, Phillips R, Frieden I, et al. Timolol maleate $0.5 \%$ or $0.1 \%$ gel-forming solution for infantile hemangiomas: a retrospective, multicenter, cohort study. Pediatr Dermatol. 2012;29(1):28-31. doi:10.1111/j.1525-1470.2011.01664.x

18. Ovadia S, Landy D, Cohen E, Yang E, Thaller S. Local administration of $\beta$-blockers for infantile hemangiomas: a systematic review and meta-analysis. Ann Plast Surg. 2015;74(2):256-262. doi:10.1097/sap.0000000000000390

19. Batta K, Goodyear H, Moss C, Williams H, Hiller L, Waters R. Randomised controlled study of early pulsed dye laser treatment of uncomplicated childhood haemangiomas: results of a 1-year analysis. Lancet. 2002;360(9332):521-527. doi:10.1016/s0140-6736(02)097 41-6

20. Chen Z, Wang Q, Zhu Y, et al. Progress in the treatment of infantile hemangioma. Ann Transl Med. 2019;7(22):692. doi:10.21037/ atm.2019.10.47

21. Ying H, Zou Y, Yu W, et al. Prospective, open-label, rater-blinded and self-controlled pilot study of the treatment of proliferating superficial infantile hemangiomas with $0.5 \%$ topical timolol cream versus 595-nm pulsed dye laser. J Dermatol. 2017;44(6):660-665. doi:10.1111/1346-8138.13747

22. Rizzo C, Brightman L, Chapas A, et al. Outcomes of childhood hemangiomas treated with the pulsed-dye laser with dynamic cooling: a retrospective chart analysis. Dermatol Surg. 2009;35 (12):1947-1954. doi:10.1111/j.1524-4725.2009.01356.x

23. Poetke M, Philipp C, Berlien H. Flashlamp-pumped pulsed dye laser for hemangiomas in infancy: treatment of superficial vs mixed hemangiomas. Arch Dermatol. 2000;136(5):628-632. doi:10.1001/ archderm.136.5.628

24. Zide B, Levine S. Hemangioma update: pearls from 30 years of treatment. Ann Plast Surg. 2012;69(1):99-103. doi:10.1097/ SAP.0b013e3182212881

25. Park K, Jang Y, Chung H, Lee W, Kim D, Lee S. Topical timolol maleate $0.5 \%$ for infantile hemangioma; it's effectiveness and/or adjunctive pulsed dye laser - single center experience of 102 cases in Korea. J Dermatolog Treat. 2015;26(4):389-391. doi:10.3109/ 09546634.2014.990412

26. Hunzeker C, Geronemus R. Treatment of superficial infantile hemangiomas of the eyelid using the 595-nm pulsed dye laser. Dermatologic surgery : official publication for American Society for Dermatologic Surgery. 2010;36(5): 590-597. doi:10.1111/j.15244725.2010.01511.x

27. Chen X, Guo Y, Wang P, et al. Efficacy and safety of adrenergic beta-antagonist combined with lasers in the treatment of infantile hemangiomas: a meta-analysis. Pediatr Surg Int. 2020;36 (10):1135-1147. doi:10.1007/s00383-020-04711-2
The manuscript management system is completely online and includes a very quick and fair peer-review system, which is all easy to use. Visit http://www.dovepress.com/testimonials.php to read real quotes from published authors. 